\title{
Fusion systems of blocks of finite groups over arbitrary fields*
}

\author{
Robert Boltje, Çisil Karagüzel, Deniz Yılmaz \\ Department of Mathematics \\ University of California \\ Santa Cruz, CA 95064 \\ U.S.A. \\ boltje@ucsc.edu, ckaraguz@ucsc.edu, deyilmaz@ucsc.edu
}

June 20, 2019

\begin{abstract}
To any block idempotent $b$ of a group algebra $k G$ of a finite group $G$ over a field $k$ of characteristic $p>0$, Puig associated a fusion system and proved that it is saturated if the $k$-algebra $k C_{G}(P) e$ is split, where $(P, e)$ is a maximal $k G b$-Brauer pair. We investigate in the non-split case how far the fusion system is from being saturated by describing it in an explicit way as being generated by the fusion system of a related block idempotent over a larger field together with a single automorphism of the defect group.
\end{abstract}

\section{Introduction}

Let $k$ be a field of characteristic $p$, let $G$ be a finite group and let $b$ be a block idempotent of $k G$. Puig defined a fusion system $\mathcal{F}_{(P, e)}(k G b)$ associated to $k G b$ after choosing a maximal $k G b$-Brauer pair $(P, e)$. Up to category isomorphism, this fusion system does not depend on the choice of $(P, e)$. Puig also proved that $\mathcal{F}_{(P, e)}(k G b)$ is saturated if the $k$-algebra $k C_{G}(P) e$ is split. It is known that in the non-split case it can happen that the fusion system associated to $k G b$ is not saturated. In fact, the Sylow axiom can fail, while the extension axiom always holds. In the Main Theorem 5.2 of this paper we establish a precise connection between the fusion systems of related blocks in a Galois extension $L / K$ of fields of characteristic $p$ with Galois group $\Gamma$. More precisely, let $b$ be a block idempotent of $L G$ and $\tilde{b}$ the unique block idempotent of $K G$ with $b \tilde{b}=b$. Moreover, let $(P, e)$ be a maximal $L G b$-Brauer pair and let $\tilde{e}$ be the unique block idempotent of $K C_{G}(P)$ with $e \tilde{e}=e$. Then $(P, \tilde{e})$ is a maximal $K G \tilde{b}$-Brauer pair and one has an inclusion of the fusion systems

$$
\mathcal{F}:=\mathcal{F}_{(P, e)}(L G b) \subseteq \mathcal{F}_{(P, \tilde{e})}(K G \tilde{b})=: \tilde{\mathcal{F}}
$$

Theorem 5.2 states that there exists an element $\sigma \in \operatorname{Aut}_{\tilde{\mathcal{F}}}(P)$ such that $\tilde{\mathcal{F}}=\langle\mathcal{F}, \sigma\rangle$. As consequences of the nature of $\sigma$ we obtain that $\tilde{\mathcal{F}}$ is saturated if and only if $\mathcal{F}$ is saturated and

*MR Subject Classification: 20C20. Keywords: Blocks of finite groups; fusion systems 
$p$ does not divide the index $\left[\Gamma_{b}: \Gamma_{e}\right]=[K(e): K(b)]$ of the stabilizers of $b$ and $e$ under the Galois action, or equivalently the degree of the field extensions after adjoining the coefficients of $e$ and $b$ to $K$. In the case that $L$ is chosen such that $L C_{G}(P) e$ is split, this gives a particularly handy criterion for a fusion system of a block $K G \tilde{b}$ in the non-split case to be saturated, see Theorem 6.3. The main result allows an alternative easy proof for the known fact that the extension axiom holds also in the non-split case, see Theorem 6.2. Finally, the Main Theorem implies that a weak form of Alperin's fusion theorem holds also for arbitrary block fusion systems, see Theorem 6.5.

1.1 Notation We will use the following standard notations without further notice:

For a group $G$ and $x \in G$, we denote by $c_{x}: G \rightarrow G$ the conjugation map $g \mapsto x g x^{-1}$. If $k$ is a commutative ring, its $k$-linear extension to the group algebra $k G$ is again denoted by $c_{x}: k G \rightarrow k G$. We frequently will use left-exponential notation ${ }^{x}(-):=c_{x}$ for these maps. The maps $c_{x}, x \in G$, define an action of $G$ on $k G$ via $k$-algebra homomorphisms.

For $H \leqslant G$, we denote by $[G / H]$ a set of representatives of the cosets $G / H$.

If a group $G$ acts on a set $X$, we usually denote the stabilizer of an element $x \in X$ by $G_{x}$. Moreover, for $H \leqslant G$, we denote by $X^{H}$ the set of $H$-fixed points of $X$.

\section{Brauer pairs}

Throughout this section, $G$ denotes a finite group, $k$ denotes a field of characteristic $p>0$, and $b$ denotes a block idempotent of $k G$, i.e., a primitive idempotent of $Z(k G)$. We recall the definition and properties of Brauer pairs for $k G$ following the treatment in AKO11, IV.2]. We note that the blanket assumption in [AKO11, IV.2] that $k$ is algebraically closed is not used in the proofs of any of the statements that we cite from there. Alternatively, see also [L18, Sections 5.9 and 6.3].

Recall that, for a $p$-subgroup $P$ of $G$, the Brauer homomorphism with respect to $P$ is the $k$-linear projection map $\operatorname{Br}_{P}:(k G)^{P} \rightarrow k C_{G}(P), \sum_{g \in G} \alpha_{g} g \mapsto \sum_{g \in C_{G}(P)} \alpha_{g} g$. This is a surjective $k$-algebra homomorphism which respects $G$-conjugation: $c_{x} \circ \operatorname{Br}_{P}=\operatorname{Br}_{P} \circ c_{x}:(k G)^{P} \rightarrow$ $k C_{G}\left({ }^{x} P\right)$ for $x \in G$. Thus, $\operatorname{Br}_{P}(b)$ is an idempotent of $Z\left(k C_{G}(P)\right)=\left(k C_{G}(P)\right)^{C_{G}(P)}$. Recall further that a $k G$-Brauer pair is a pair $(P, e)$ consisting of a $p$-subgroup $P$ of $G$ and a block idempotent $e$ of $k C_{G}(P)$. If $e$ occurs in the unique decomposition of $\operatorname{Br}_{P}(b)$ into a sum of primitive idempotents of $Z\left(k C_{G}(P)\right)$ (that is, if $\left.\operatorname{Br}_{P}(b) e=e\right)$, then we call $(P, e)$ a $(k G, b)$ Brauer pair. We denote by $\mathcal{B P}(k G)$ the set of $k G$-Brauer pairs and by $\mathcal{B P}(k G, b)$ the set of $(k G, b)$-Brauer pairs. Clearly, $\mathcal{B P}(k G)$ is the disjoint union of the subsets $\mathcal{B P}(k G, b)$, where $b$ runs through the block idempotents of $k G$. The set $\mathcal{B P}(k G)$ is a $G$-set under the conjugation action given by ${ }^{x}(P, e):=\left({ }^{x} P,{ }^{x} e\right)$, and the subset $\mathcal{B P}(k G, b)$ is $G$-stable. Finally, we say that an idempotent $i$ of $(k G)^{P}$ is associated to a $k G$-Brauer pair $(P, e)$ if

$$
e \operatorname{Br}_{P}(i)=\operatorname{Br}_{P}(i) \neq 0 .
$$

Note that if $i$ is primitive in $(k G)^{P}$ then $e \operatorname{Br}_{P}(i) \neq 0$ implies that $\operatorname{Br}_{P}(i) \neq 0$ and that $\operatorname{Br}_{P}(i)$ is primitive in $k C_{G}(P)$. Thus, $e \operatorname{Br}_{P}(i)=\operatorname{Br}_{P}(i)$. One writes $(Q, f) \leqslant(P, e)$ if $Q \leqslant P$ and if any primitive idempotent $i$ of $(k G)^{P}$ which is associated to $(P, e)$ is also associated to $(Q, f)$, see [AKO11, Definition 2.9]. This relation has the following properties. 
2.1 Theorem (AKO11, Theorems 2.10, 2.16]) (a) Let $(P, e) \in \mathcal{B P}(k G)$ and let $Q \leqslant P$. Then there exists a unique block idempotent $f$ of $k C_{G}(Q)$ such that $(Q, f) \leqslant(P, e)$.

(b) Let $(Q, f) \leqslant(P, e)$ be in $\mathcal{B P}(k G)$ with $Q \unlhd P$. Then $f$ is the unique block idempotent of $k C_{G}(Q)$ which is $P$-stable and satisfies $B r_{P}(f) e=e$.

(c) The relation $\leqslant$ on $\mathcal{B P}(k G)$ is a partial order which is respected by the conjugation action of $G$.

Clearly $(\{1\}, b) \in \mathcal{B P}(k G, b)$ and Part (b) of the above theorem implies that if $(P, e) \in$ $\mathcal{B P}(k G, b)$ then $(\{1\}, b) \leqslant(P, e)$. Parts (a) and (c) further imply that if $(Q, f) \leqslant(P, e)$ holds for elements in $\mathcal{B P}(k G)$ then $(Q, f) \in \mathcal{B P}(k G, b)$ if and only if $(P, e) \in \mathcal{B P}(k G, b)$.

For Brauer pairs $(Q, f),(P, e) \in \mathcal{B P}(k G)$ one writes $(Q, f) \unlhd(P, e)$ if $Q \unlhd P, f$ is $P$ stable and $\operatorname{Br}_{P}(f) e=e$, cf. [AKO11, Definition IV.2.13]. The following result is well-known to specialists. We state it for convenient future reference and give a proof for the convenience of the reader.

2.2 Theorem For $(Q, f),(P, e) \in \mathcal{B P}(k G)$ with $Q \leqslant P$ the following statements are equivalent:

(i) One has $(Q, f) \leqslant(P, e)$.

(ii) There exist primitive idempotents $i$ of $(k G)^{P}$ and $j$ of $(k G)^{Q}$ such that $i j=j=j i$, $\operatorname{Br}_{P}(i) e \neq 0$ and $\operatorname{Br}_{Q}(j) f \neq 0$.

(iii) There exist Brauer pairs $\left(Q_{i}, d_{i}\right) \in \mathcal{B P}(k G), i=0, \ldots, n$, such that

$$
(Q, f)=\left(Q_{0}, d_{0}\right) \unlhd\left(Q_{1}, d_{1}\right) \unlhd \cdots \unlhd\left(Q_{n}, d_{n}\right)=(P, e) .
$$

(iv) For every primitive idempotent $i$ of $(k G)^{P}$ with $B r_{P}(i) e \neq 0$ one has $B r_{Q}(i) f \neq 0$.

(v) There exists a primitive idempotent $i$ of $(k G)^{P}$ such that $\operatorname{Br}_{P}(i) e \neq 0$ and $\operatorname{Br}_{Q}(i) f=$ $\operatorname{Br}_{Q}(i) \neq 0$.

(vi) There exists a primitive idempotent $i$ of $(k G)^{P}$ such that $B r_{P}(i) e \neq 0$ and $\operatorname{Br}_{Q}(i) f \neq 0$.

Proof The equivalences (i) $\Longleftrightarrow$ (ii) $\Longleftrightarrow$ (iii) follow from [AKO11, Proposition IV.2.14]. Moreover, the implications (i) $\Rightarrow$ (iv) and (v) $\Rightarrow$ (vi) are trivial and the implication (i) $\Rightarrow(v)$ follows from the fact that the image of a primitive idempotent under a surjective $k$-algebra homomorphism is either 0 or a primitive idempotent.

Next we show that (iv) implies (i). Let $i$ be a primitive idempotent of $(k G)^{P}$ such that $\operatorname{Br}_{P}(i) e=\operatorname{Br}_{P}(i) \neq 0$. By (iv), $\operatorname{Br}_{Q}(i) f \neq 0$. By Theorem 2.1(a) there exists a block idempotent $f^{\prime}$ of $k C_{G}(Q)$ such that $\left(Q, f^{\prime}\right) \leqslant(P, e)$. Thus, $\operatorname{Br}_{Q}(i) f^{\prime}=B r_{Q}(i)$ which implies that $0 \neq$ $\operatorname{Br}_{Q}(i) f=\operatorname{Br}_{Q}(i) f^{\prime} f$ and further that $f=f^{\prime}$ and thus $(Q, f) \leqslant(P, e)$.

Finally, we show that (vi) implies (i). Let $i$ be as in (vi). By Theorem 2.1(a) there exists a block idempotent $f^{\prime}$ of $k C_{G}(Q)$ such that $\left(Q, f^{\prime}\right) \leqslant(P, e)$. This implies $\operatorname{Br}_{Q}(i) f^{\prime}=\operatorname{Br}_{Q}(i) \neq 0$ and $0 \neq \mathrm{Br}_{Q}(i) f=\operatorname{Br}_{Q}(i) f^{\prime} f$. Thus $f=f^{\prime}$ and $(Q, f) \leqslant(P, e)$.

Recall that if $I \leqslant H \leqslant G$ then we have a well-defined trace map

$$
\operatorname{Tr}_{I}^{H}:(k G)^{I} \rightarrow(k G)^{H}, \quad a \mapsto \sum_{x \in[H / I]} x_{a} .
$$

A subgroup $P$ of $G$, minimal with the property that $b \in \operatorname{Tr}_{P}^{G}\left((k G)^{P}\right)$, is called a defect group of the block idempotent $b$ and of the block algebra $k G b$. The defect groups of $k G b$ form a single 
$G$-conjugacy class of $p$-subgroups of $G$. Maximal elements in $\mathcal{B P}(k G, b)$ enjoy properties that resemble the Sylow Theorem for finite groups.

2.3 Theorem ([AKO11, Theorem 2.20]) (a) The maximal elements in $\mathcal{B P}(k G, b)$ with respect to $\leqslant$ form a single $G$-orbit.

(b) For $(P, e) \in \mathcal{B P}(k G, b)$ the following are equivalent.

(i) $(P, e)$ is a maximal element in $\mathcal{B P}(k G, b)$.

(ii) $P$ is a defect group of $k G b$.

(iii) $P$ is maximal among all $p$-subgroups of $G$ with the property $\operatorname{Br}_{P}(b) \neq 0$.

\section{$3 \quad$ Fusion systems of block algebras}

Throughout this section, $p$ is a prime. We first recall the basic notions and properties of fusion systems, a structure introduced by Puig. Our terminology follows [AKO11, Chapter I].

For subgroups $Q$ and $R$ of a finite group $G$ we denote by $\operatorname{Hom}_{G}(Q, R)$ the set of all group homomorphisms $\varphi: Q \rightarrow R$ with the property that there exists $x \in G$ with $\varphi(u)=c_{x}(u)$ for all $u \in Q$. Moreover, we set $\operatorname{Aut}_{G}(Q):=\operatorname{Hom}_{G}(Q, Q)$.

3.1 Definition ([AKO11, Definition I.2.1]) Let $P$ be a finite $p$-group. A subcategory $\mathcal{F}$ of the category of finite groups whose objects are the subgroups of $P$ is called a fusion system over $P$ if for any two subgroups $Q$ and $R$ of $P$, the set $\operatorname{Hom}_{\mathcal{F}}(Q, R)$ has the following properties:

(i) $\operatorname{Hom}_{P}(Q, R) \subseteq \operatorname{Hom}_{\mathcal{F}}(Q, R)$ and every element of $\operatorname{Hom}_{\mathcal{F}}(Q, R)$ is injective.

(ii) For each $\varphi \in \operatorname{Hom}_{\mathcal{F}}(Q, R)$, the group isomorphism $Q \rightarrow \varphi(Q), u \mapsto \varphi(u)$, and its inverse are morphisms in $\mathcal{F}$.

For instance, if $G$ is a finite group and $P$ is a $p$-subgroup of $G$, we obtain a fusion system $\mathcal{F}_{P}(G)$ over $P$ by setting $\operatorname{Hom}_{\mathcal{F}_{P}(G)}(Q, R):=\operatorname{Hom}_{G}(Q, R)$, for all subgroups $Q$ and $R$ of $P$. Note that the intersection of two fusion systems over $P$ is again a fusion system and that a fusion system over $P$ is determined by the isomorphisms it contains. Thus the smallest fusion system over a finite $p$-group $P$ is the fusion system $\mathcal{F}_{P}(P)$.

3.2 Definition ([AKO11, Definition I.2.4]) Let $\mathcal{F}$ be a fusion system over a finite $p$-group $P$. A subgroup $Q$ of $P$ is called fully $\mathcal{F}$-centralized if $\left|C_{P}(Q)\right| \geqslant\left|C_{P}\left(Q^{\prime}\right)\right|$ for any subgroup $Q^{\prime}$ of $P$ which is $\mathcal{F}$-isomorphic to $Q$. Similarly, $Q$ is called fully $\mathcal{F}$-normalized if $\left|N_{P}(Q)\right| \geqslant\left|N_{P}\left(Q^{\prime}\right)\right|$ for any subgroup $Q^{\prime}$ of $P$ which is $\mathcal{F}$-isomorphic to $Q$.

3.3 Definition ([AKO11, Definition I.2.2]) Let $\mathcal{F}$ be a fusion system on a finite $p$-group $P$ and let $\varphi: Q \rightarrow R$ be an isomorphism in $\mathcal{F}$. One denotes by $N_{\varphi}$ the set of all elements $y \in N_{P}(Q)$ for which there exists $z \in N_{P}(R)$ with the property $\varphi \circ c_{y}=c_{z} \circ \varphi: Q \rightarrow R$. Note that $Q C_{P}(Q) \leqslant N_{\varphi} \leqslant N_{P}(Q)$ and that $N_{\varphi}$ does not depend on $\mathcal{F}$, but only on $\varphi$ and $P$.

If $\mathcal{F}$ is a fusion system over a finite $p$-group $P$ and $Q \leqslant P$ then we set $\operatorname{Aut}_{\mathcal{F}}(Q):=$ $\operatorname{Hom}_{\mathcal{F}}(Q, Q)$, a subgroup of the automorphism group of $Q$. The following definition of saturation goes back to Stancu and is an equivalent reformulation of the original definition, see [AKO11, Proposition I.9.3]. 
3.4 Definition A fusion system $\mathcal{F}$ over a $p$-group $P$ is called saturated if the following two conditions hold.

(i) Sylow axiom: The group $\operatorname{Aut}_{P}(P)$ is a Sylow $p$-subgroup of $\operatorname{Aut}_{\mathcal{F}}(P)$.

(ii) Extension axiom: For every $Q \leqslant P$ and every $\varphi \in \operatorname{Hom}_{\mathcal{F}}(Q, P)$ such that $\varphi(Q)$ is fully $\mathcal{F}$-normalized there exists a morphism $\psi \in \operatorname{Hom}_{\mathcal{F}}\left(N_{\varphi}, P\right)$ whose restriction to $Q$ equals $\varphi$.

For instance, if $P$ is a Sylow $p$-subgroup of a finite group $G$ then the fusion system $\mathcal{F}_{P}(G)$ is saturated (see [AKO11, Theorem 2.3]).

3.5 Definition Let $G$ be a finite group, let $k$ be a field of characteristic $p$, let $b$ be a block idempotent of $k G$, and let $(P, e)$ be a maximal $(k G, b)$-Brauer pair. We define a category $\mathcal{F}_{(P, e)}(k G b)$ as follows. First, for every $Q \leqslant P$ denote by $e_{Q}$ the unique block idempotent of $k C_{G}(Q)$ with $\left(Q, e_{Q}\right) \leqslant(P, e)$. The objects of $\mathcal{F}_{(P, e)}(k G b)$ are the subgroups of $P$ and for subgroups $Q$ and $R$ of $P$ let $\operatorname{Hom}_{\mathcal{F}_{(P, e)}(k G b)}(Q, R)$ denote the set of group homomorphisms $\varphi: Q \rightarrow R$ such that there exists $x \in G$ with $\varphi(u)=c_{x}(u)$ for all $u \in Q$ and ${ }^{x}\left(Q, e_{Q}\right) \leqslant\left(R, e_{R}\right)$. Composition in $\mathcal{F}_{(P, e)}(k G b)$ is the usual composition of functions.

3.6 Remark Let $k G, b$, and $(P, e)$ be as in Definition 3.5.

(a) It is clear from the definition that $\mathcal{F}_{(P, e)}(k G b)$ is a fusion system over $P$.

(b) If $k G b$ is the principal block of $k G$, then by Brauer's third main theorem, $\mathcal{F}_{(P, e)}(k G b)$ is equal to $\mathcal{F}_{P}(G)$ and $P$ is a Sylow $p$-subgroup of $G$. Thus, $\mathcal{F}_{(P, e)}(k G b)$ is saturated in this case.

(c) Example 3.8 below shows that in general the Sylow axiom does not hold for $\mathcal{F}_{(P, e)}(k G b)$. But we will show in Theorem 6.2 that the extension axiom holds for $\mathcal{F}_{(P, e)}(k G b)$.

The following theorem was first proved by Puig. It follows from Theorem IV.3.2 and Proposition IV.3.14 in [AKO11]. See also [L18, Theorem 8.5.2] and note that there the terminology is different: Fusion systems in [L18] are defined to be saturated fusion systems in our terminology.

3.7 Theorem Let $k G, b$, and $(P, e)$ be as in Definition 3.5 and suppose that the $k$-algebra $k C_{G}(P) e$ is split, i.e., for every simple $k C_{G}(P)$ e-module $V$ one has a $k$-algebra isomorphism $\operatorname{End}_{k C_{G}(P) e}(V) \cong k$. Then the fusion system $\mathcal{F}_{(P, e)}(k G b)$ is saturated.

We are grateful to Radha Kessar who suggested the following example to us.

3.8 Example Let $p=2, k=\mathbb{F}_{2}$, the field with 2 elements, and $G:=D_{24}=\left(C_{3} \times C_{4}\right) \rtimes C_{2}$, the dihedral group with 24 elements, with $C_{2}$ acting by inversion on $C_{3} \times C_{4}$. Let $g$ denote a generator of $C_{3}$. Then $b:=g+g^{2}$ is a block idempotent of $\mathbb{F}_{2} G$ and $(P, e):=\left(C_{4}, b\right)$ is a maximal $\left(\mathbb{F}_{2} G, b\right)$-Brauer pair. We have $\operatorname{Aut}_{P}(P)=\{1\}$, since $P$ is abelian and an easy computation shows that $\operatorname{Aut}_{\mathcal{F}_{(P, e)}\left(\mathbb{F}_{2} G b\right)}(P) \cong C_{2}$. Thus, the Sylow axiom does not hold for $\mathcal{F}_{(P, e)}\left(\mathbb{F}_{2} G b\right)$ and therefore the fusion system $\mathcal{F}_{(P, e)}\left(\mathbb{F}_{2} G b\right)$ is not saturated.

\section{Extension of scalars}

Throughout this section $L / K$ denotes a finite Galois extension of fields of characteristic $p>0$ and $\Gamma$ denotes its Galois group. Moreover, $G$ denotes a finite group.

$\Gamma$ acts via $K$-algebra automorphisms on the group algebra $L G$ and also on $Z(L G)$ by applying $\gamma \in \Gamma$ to the the coefficients of an element in $L G$. Thus, $\Gamma$ permutes the block idempotents of $L G$ and fixes the block idempotents $b$ of $K G$. Since $\operatorname{Br}_{P}:(L G)^{P} \rightarrow L C_{G}(P)$ 
commutes with the $\Gamma$-action, Theorem 2.3 implies that any $\Gamma$-conjugate of $b$ has the same defect groups as $b$. We denote by $\Gamma_{b}$ the stabilizer of $b$ in $\Gamma$ and set

$$
\tilde{b}:=\sum_{\gamma \in\left[\Gamma / \Gamma_{b}\right]} \gamma_{b}
$$

Clearly, $\tilde{b}$ is an idempotent in $(Z(L G))^{\Gamma}=Z(K G)$. More precisely one has the following:

4.1 Proposition (a) Let $b$ be a block idempotent of $L G$. Then $\tilde{b}:=\sum_{\gamma \in\left[\Gamma / \Gamma_{b}\right]} \gamma_{b}$ is a block idempotent of $K G$.

(b) The map $b \mapsto \tilde{b}$ induces a bijection between the set of $\Gamma$-orbits of block idempotents of $L G$ and the set of block idempotents of $K G$.

(c) If $b$ is a block idempotent of $L G$ and $\tilde{b}$ is the block idempotent of $K G$ associated to it as in (a) then $b$ and $\tilde{b}$ have the same defect groups.

Proof (a) By definition, $\tilde{b}$ is the sum of the distinct $\Gamma$-conjugates of $b$, thus an idempotent of $Z(K G)$. To see that $\tilde{b}$ is primitive in $Z(K G)$, assume that $\tilde{b}=c_{1}+c_{2}$ for non-zero orthogonal idempotents $c_{1}, c_{2} \in Z(K G)$ and let $I_{1}$ and $I_{2}$ denote the set of primitive idempotents of $Z(L G)$ that occur in a primitive decomposition of $c_{1}$ and $c_{2}$ in $Z(L G)$, respectively. Then $I_{1}$ and $I_{2}$ are disjoint and $\Gamma$-stable. On the other hand $I_{1} \cup I_{2}$ is the single $\Gamma$-orbit of $b$. This is a contradiction.

(b) This is immediate from (a).

(c) Let $P$ be a defect group of $\tilde{b}$. By Theorem 2.3, one has $\operatorname{Br}_{P}(\tilde{b}) \neq 0$ in $K C_{G}(P) \subseteq L C_{G}(P)$. Thus $0 \neq \operatorname{Br}_{P}(\tilde{b})=\sum_{\gamma \in\left[\Gamma / \Gamma_{b}\right]} \operatorname{Br}_{P}(\gamma b)$ implies that some $\Gamma$-conjugate of $b$, and therefore also $b$, has a defect group $Q$ containing $P$. Thus, $0 \neq \operatorname{Br}_{Q}(b)=\operatorname{Br}_{Q}(b \tilde{b})=\operatorname{Br}_{Q}(b) \operatorname{Br}_{Q}(\tilde{b})$, which implies that $\operatorname{Br}_{Q}(\tilde{b}) \neq 0$ and therefore $|Q| \leqslant|P|$. This implies $P=Q$.

Note that $\Gamma$ acts on $\mathcal{B P}(L G)$ via

$$
{ }^{\gamma}(P, e)=\left(P,{ }^{\gamma} e\right)
$$

for $\gamma \in \Gamma$ and $(P, e) \in \mathcal{B P}(L G)$. Note that this action commutes with the $G$-action on $\mathcal{B P}(L G)$ so that we obtain an action of $\Gamma \times G$ on $\mathcal{B P}(L G)$. Moreover, since $\operatorname{Br}_{P}$ commutes with the action of $\Gamma$ and since the $G$-action on $L G$ commutes with the $\Gamma$-action on $L G, \Gamma \times G$ acts via poset isomorphisms on $\mathcal{B P}(L G)$. Thus, if $b$ is a block idempotent of $L G$ and $\gamma \in \Gamma$, the $G$-posets $\mathcal{B P}(L G b)$ and $\mathcal{B P}\left(L G^{\gamma} b\right)$ are isomorphic via (1) and $\Gamma_{b} \times G$ acts via poset automorphisms on $\mathcal{B P}(L G b)$.

In the next proposition we write $\leqslant_{K}$ and $\leqslant_{L}$ for the poset structures of $\mathcal{B P}(K G)$ and $\mathcal{B P}(L G)$, respectively. They are related as follows.

4.2 Proposition For $(Q, f),(P, e) \in \mathcal{B P}(L G)$ with $Q \leqslant P$, the following are equivalent:

(i) One has $(Q, \tilde{f}) \leqslant_{K}(P, \tilde{e})$ in $\mathcal{B P}(K G)$.

(ii) There exists $\gamma \in \Gamma$ such that $(Q, f) \leqslant{ }_{L}^{\gamma}(P, e)$ in $\mathcal{B P}(L G)$.

Proof Assume first that (i) holds and let $i$ be a primitive idempotent of $(K G)^{P}$ such that $\operatorname{Br}_{P}(i) \tilde{e}=\operatorname{Br}_{P}(i) \neq 0$. Then, by definition also $\operatorname{Br}_{Q}(i) \tilde{f}=\operatorname{Br}_{Q}(i) \neq 0$. Let $J$ be a primitive decomposition of $i$ in $(L G)^{P}$. Since $\operatorname{Br}_{P}(i) \tilde{e} \neq 0$, there exists $j \in J$ such that $\operatorname{Br}_{P}(j) \tilde{e} \neq 0$. Thus, there exists $\gamma \in \Gamma$ such that $\operatorname{Br}_{P}(j)^{\gamma} e \neq 0$. Since $\operatorname{Br}_{P}(j)$ is primitive in $L C_{G}(P)$, we have $\operatorname{Br}_{P}(j)^{\gamma} e=\operatorname{Br}_{P}(j)$. Let $f^{\prime}$ be the block idempotent of $L C_{G}(Q)$ such that $\left(Q, f^{\prime}\right) \leqslant L$ 
$\left(P,{ }^{\gamma} e\right)={ }^{\gamma}(P, e)$. Then, by Theorem 2.2 also $\operatorname{Br}_{Q}(j) f^{\prime}=\operatorname{Br}_{Q}(j) \neq 0$. Thus $\operatorname{Br}_{Q}(j) f^{\prime} \tilde{f}=$ $\operatorname{Br}_{Q}(j) \operatorname{Br}_{Q}(i) f^{\prime} f=\operatorname{Br}_{Q}(j) f^{\prime} \operatorname{Br}_{Q}(i) \tilde{f}=\operatorname{Br}_{Q}(j) \operatorname{Br}_{Q}(i)=\operatorname{Br}_{Q}(j) \neq 0$ which implies that $f^{\prime} \tilde{f} \neq 0$. This implies $f^{\prime}={ }^{\delta} f$ for some $\delta \in \Gamma$. Thus ${ }^{\delta}(Q, f) \leqslant{ }_{L}{ }^{\gamma}(P, e)$ and (ii) holds after applying $\delta^{-1}$.

Next assume that $\gamma \in \Gamma$ with $(Q, f) \leqslant_{L}{ }^{\gamma}(P, e)$. By Theorem 2.1(a) there exists a block idempotent $f_{1}$ of $L C_{G}(Q)$ such that $\left(Q, \tilde{f}_{1}\right) \leqslant_{K}(P, \tilde{e})$. Since we already proved that (i) implies (ii), there exists $\delta \in \Gamma$ such that $\left(Q, f_{1}\right) \leqslant_{L}{ }^{\delta}(P, e)$. Thus we have $\left(Q, \gamma^{-1} f\right) \leqslant_{L}(P, e)$ and also $\left(Q,{ }^{-1} f\right) \leqslant_{L}(P, e)$. The uniqueness part of Theorem 2.2(a) now implies that $f$ and $f_{1}$ are $\Gamma$-conjugate. Thus $\tilde{f}=\tilde{f}_{1}$ and $(Q, \tilde{f}) \leqslant_{K}(P, \tilde{e})$.

The following corollaries are now immediate from Proposition 4.2 .

\subsection{Corollary The map}

$$
\mathcal{B P}(L G) \rightarrow \mathcal{B P}(K G), \quad(P, e) \mapsto(P, \tilde{e}),
$$

is a surjective morphism of $G$-posets, which restricts to a surjective morphism of $G$-posets $\mathcal{B P}(L G b) \rightarrow \mathcal{B P}(K G \tilde{b})$ for every block idempotent $b$ of $L G$.

4.4 Corollary Let $b$ be a block idempotent of $L G$ and let $(P, e) \in \mathcal{B P}(L G b)$ be a maximal $L G b$-Brauer pair. Then $(P, \tilde{e}) \in \mathcal{B P}(K G \tilde{b})$ is a maximal $(K G \tilde{b})$-Brauer pair and one obtains an inclusion of fusion systems

$$
\mathcal{F}_{(P, e)}(L G b) \rightarrow \mathcal{F}_{(P, \tilde{e})}(K G \tilde{b})
$$

which is the identity on objects and on morphisms.

\section{The Main Theorem}

We keep $p, G, L / K$, and $\Gamma$ as introduced at the beginning of Section 4 . Moreover we fix a block idempotent $b$ of $L G$ and denote by $\Gamma_{b}$ the stabilizer of $b$ in $\Gamma$. We fix a maximal $L G b$-Brauer pair $(P, e) \in \mathcal{B P}(L G b)$. For every $Q \leqslant P$, let $e_{Q}$ denote the unique block idempotent of $L C_{G}(Q)$ such that $\left(Q, e_{Q}\right) \leqslant(P, e)$ in $\mathcal{B P}(L G)$. By Proposition 4.2, one has $\left(Q, \widetilde{e_{Q}}\right) \leqslant(P, \tilde{e})$ so that $\widetilde{e_{Q}}=\tilde{e}_{Q}$. This allows us to use the notation $\tilde{e}_{Q}$ for both purposes. Recall that $\Gamma \times G$ acts on $\mathcal{B P}(L G)$ and $\Gamma_{b} \times G$ acts on $\mathcal{B P}(L G b)$ via poset isomorphisms. Note that for any $(Q, f) \in \mathcal{B P}(L G b)$ one has $\Gamma_{(Q, f)}=\Gamma_{f}$. For the stabilizer in $G$ of a $K G$-Brauer pair or $L G$-Brauer pair $(Q, f)$ we will write $N_{G}(Q, f)$.

Let $p_{1}: G \times \Gamma \rightarrow G$ and $p_{2}: G \times \Gamma \rightarrow \Gamma$ denote the projection maps. For any subgroup $X$ of $G \times \Gamma$, we set $k_{1}(X):=\{g \in G \mid(g, 1) \in X\}$ and $k_{2}(X):=\{\gamma \in \Gamma \mid(1, \gamma) \in X\}$. As explained in [B10, p. 24], one has

$$
k_{1}(X) \unlhd p_{1}(X) \leqslant G \quad \text { and } \quad k_{2}(X) \unlhd p_{2}(X) \leqslant \Gamma \quad \text { with } \quad p_{1}(X) / k_{1}(X) \cong p_{2}(X) / k_{2}(X)
$$

via $g k_{1}(X) \leftrightarrow \gamma k_{2}(X)$ if and only if $(g, \gamma) \in X$.

We denote by $K(b)$ and $K(e)$ the subfields of $L$ obtained by adjoining the coefficients of the block idempotents $b \in L G$ and $e \in L C_{G}(P)$. Thus, $K(b)$ is the fixed field of $\Gamma_{b}$ in $L$ and $K(e)$ is the fixed field of $\Gamma_{e}$ in $L$. 
5.1 Proposition Let $b$ be a block idempotent of $L G$.

(a) For any $\left(R, e_{R}\right) \leqslant\left(Q, e_{Q}\right)$ in $\mathcal{B P}(L G b)$ one has $\Gamma_{e}=\Gamma_{(P, e)} \leqslant \Gamma_{\left(Q, e_{Q}\right)} \leqslant \Gamma_{\left(R, e_{R}\right)} \leqslant$ $\Gamma_{(\{1\}, b)}=\Gamma_{b}$. In particular, $K(b) \subseteq K(e)$.

(b) Let $X:=\operatorname{stab}_{G \times \Gamma}(P, e)$ be the stabilizer of the maximal LGb-Brauer pair $(P, e)$. One has

$$
k_{1}(X)=N_{G}(P, e), \quad p_{1}(X)=N_{G}(P, \tilde{e}), \quad k_{2}(X)=\Gamma_{e}, \quad \text { and } \quad p_{2}(X)=\Gamma_{b} .
$$

(c) One has $N_{G}(P, e) \unlhd N_{G}(P, \tilde{e})$ and $N_{G}(P, \tilde{e}) / N_{G}(P, e) \cong \Gamma_{b} / \Gamma_{e}$. Moreover, $K(e) / K(b)$ is a Galois extension with cyclic Galois group isomorphic to $N_{G}(P, \tilde{e}) / N_{G}(P, e)$.

Proof (a) It suffices to show that $\Gamma_{\left(Q, e_{Q}\right)} \leqslant \Gamma_{\left(R, e_{R}\right)}$. Let $\gamma \in \Gamma_{\left(Q, e_{Q}\right)}$. Then ${ }^{\gamma}\left(R, e_{R}\right) \leqslant L$ ${ }^{\gamma}\left(Q, e_{Q}\right)=\left(Q, \gamma_{e_{Q}}\right)=\left(Q, e_{Q}\right)$. The uniqueness part of Theorem 2.1(a) implies that ${ }^{\gamma} e_{R}=e_{R}$. Thus, $\gamma \in \Gamma_{\left(R, e_{R}\right)}$.

(b) The first equation is clear from the definition of $k_{1}(X)$. For the proof of the second equation, let $g \in p_{1}(X)$. Then there exists $\gamma \in \Gamma$ with $(P, e)={ }^{(g, \gamma)}(P, e)=\left({ }^{g} P,{ }^{g \gamma} e\right)$. From ${ }^{g \gamma} e=e$ it follows that ${ }^{g} \tilde{e}=\tilde{e}$. Thus ${ }^{g}(P, \tilde{e})=(P, \tilde{e})$ and $g \in N_{G}(P, \tilde{e})$. Conversely, if $g \in$ $N_{G}(P, \tilde{e})$ then ${ }^{g} \tilde{e}=\tilde{e}$ which implies that there exists $\gamma \in \Gamma$ with ${ }^{g} e=\gamma_{e}$. Thus, ${ }^{\left(g, \gamma^{-1}\right.}(P, e)=$ $(P, e)$ and $g \in p_{1}(X)$. The third equation follows immediately from the definition of $k_{2}(X)$. For the proof of the fourth equation let $\gamma \in p_{2}(X)$. Then there exists $g \in G$ with ${ }^{(g, \gamma)}(P, e)=(P, e)$. Since $(\{1\}, b) \leqslant(P, e)$, this implies ${ }^{(g, \gamma)}(\{1\}, b) \leqslant{ }^{(g, \gamma)}(P, e)=(P, e)$. The uniqueness part in Theorem 2.1 (a) implies that ${ }^{(g, \gamma)}(\{1\}, b)=(1, b)$ and that $\gamma \in \Gamma_{b}$. Conversely, assume that $\gamma \in \Gamma_{b}$. Then $(\{1\}, b) \leqslant(P, e)$ implies $(\{1\}, b)={ }^{(1, \gamma)}(\{1\}, b) \leqslant{ }^{(1, \gamma)}(P, e)=\left(P, \gamma^{\gamma}\right)$. This implies that both $(P, e)$ and ${ }^{\gamma}(P, e)$ are maximal $L G b$-Brauer pairs. By Theorem 2.3 (a), there exists $g \in G$ such that ${ }^{g}\left(P,{ }^{\gamma} e\right)=(P, e)$. Thus $(g, \gamma) \in X$ and $\gamma \in p_{2}(X)$.

(c) The assertions of the first sentence follow from Part (b) and (2). For the second statement it suffices to show that $\Gamma_{b} / \Gamma_{e}$ is cyclic. Note that the coefficients of $e \in L C_{G}(P)$ generate a finite field extension of the prime field $\mathbb{F}_{p}$ in $L$, which we denote by $\mathbb{F}_{p}(e)$. Since $\Gamma_{e} \unlhd \Gamma_{b}$, we have a Galois extension $K(e) / K(b)$ with Galois group $\Delta \cong \Gamma_{b} / \Gamma_{e}$. Now, restriction from $K(e)$ to $\mathbb{F}_{p}(e)$ is an injective group homomorphism from $\Delta$ to the cyclic Galois group $\operatorname{Gal}\left(\mathbb{F}_{p}(e) / \mathbb{F}_{p}\right)$. In fact, if $\delta \in \Delta$ restricts to the identity on $\mathbb{F}_{p}(e)$, then it is the identity on $\mathbb{F}_{p}(e)$ and on $K$, thus on $K(e)$. This completes the proof of Part (c).

Next we give a more precise picture of the inclusion of fusion systems from Corollary 4.4. In the following theorem the term $\langle\mathcal{F}, \sigma\rangle$ denotes the fusion system generated by $\mathcal{F}$ and $\sigma$, i.e., the intersection of all fusion systems over $P$ that contain $\mathcal{F}$ and $\sigma$.

5.2 Theorem Let $L / K$ be a finite Galois extension of fields of characteristic $p>0$ with Galois group $\Gamma$, let $b$ be a block idempotent of $L G$, and let $(P, e)$ be a maximal $L G b$-Brauer pair. Set $\mathcal{F}:=\mathcal{F}_{(P, e)}(L G b)$ and $\tilde{\mathcal{F}}:=\mathcal{F}_{(P, \tilde{e})}(K G \tilde{b})$. Let $g_{0} \in N_{G}(P, e)$ be such that $g_{0} N_{G}(P, \tilde{e})$ generates $N_{G}(P, e) / N_{G}(P, \tilde{e})$ (see Proposition [5.1 $(c)$ ) and set $\sigma:=c_{g_{0}} \in \operatorname{Aut}(P)$. Then $\tilde{\mathcal{F}}=\langle\mathcal{F}, \sigma\rangle$.

More precisely, $\sigma \in \operatorname{Aut}_{\tilde{\mathcal{F}}}(P)$ and, for any subgroups $Q$ and $R$ of $P$ and any $\varphi \in$ $\operatorname{Hom}_{\tilde{\mathcal{F}}}(Q, R)$, there exist $i \in \mathbb{Z}, \psi \in \operatorname{Hom}_{\mathcal{F}}\left(Q, \sigma^{-i}(R)\right)$ and $\psi^{\prime} \in \operatorname{Hom}_{\mathcal{F}}\left(\sigma^{i}(Q), R\right)$ with $\varphi=\left.\sigma^{i}\right|_{\sigma^{-i}(R)} \circ \psi=\left.\psi^{\prime} \circ \sigma^{i}\right|_{Q}$.

Proof Since $g_{0} \in N_{G}(P, \tilde{e})$, we have $\sigma=c_{g_{0}} \in \operatorname{Aut}_{\tilde{\mathcal{F}}}(P)$. It follows that $\langle\mathcal{F}, \sigma\rangle \subseteq \tilde{\mathcal{F}}$. In order to prove the reverse inclusion, let $Q$ and $R$ be subgroups of $P$ and let $\varphi \in \operatorname{Hom}_{\tilde{\mathcal{F}}}(Q, R)$. Then there exists $g \in G$ such that $\varphi=c_{g}: Q \rightarrow R$ and ${ }^{g}\left(Q, \tilde{e}_{Q}\right) \leqslant K\left(R, \tilde{e}_{R}\right)$. By Proposition 4.2 
there exists $\gamma \in \Gamma$ such that ${ }^{g}\left(Q, e_{Q}\right) \leqslant{ }_{L}\left(R,{ }^{\gamma} e_{R}\right)$. Since $(\{1\}, b)={ }^{g}(\{1\}, b) \leqslant{ }_{L}{ }^{g}\left(Q, e_{Q}\right) \leqslant{ }_{L}$ $\left(R, \gamma^{\gamma} e_{R}\right)$ and also $(\{1\}, \gamma b) \leqslant_{L}\left(R, \gamma^{\gamma} e_{R}\right)$, Theorem $2.1(\mathrm{a})$ implies $(\{1\}, b)=\left(\{1\}, \gamma^{\gamma} b\right)$ so that $\gamma \in \Gamma_{b}$. Thus, both $(P, e)$ and $\left(P, \gamma^{\gamma}\right)$ are maximal $L G b$-Brauer pairs. Theorem [2.3)(a) implies that there exists $h \in G$ such that ${ }^{h}(P, e)=\left(P, \gamma_{e}\right)$ and we obtain $(P, e)={ }^{h^{-1}}\left(P, \gamma_{e}\right) \geqslant_{L}$ ${ }^{h^{-1}}\left(R,{ }^{\gamma} e_{R}\right)=\left({ }^{h^{-1}} R,{ }^{h^{-1} \gamma} e_{R}\right)$. Again, Theorem 2.1(a) implies that ${ }^{h^{-1} \gamma} e_{R}=e_{h^{-1} R h}$ and therefore ${ }^{h^{-1} g}\left(Q, e_{Q}\right) \leqslant{ }_{L}{ }^{h^{-1}}\left(R,{ }^{\gamma} e_{R}\right)=\left({ }^{h^{-1}} R, e_{h^{-1} R h}\right)$. This in turn implies that the homomorphism $\alpha:=c_{h^{-1} g}: Q \rightarrow{ }^{h^{-1}} R$ belongs to $\operatorname{Hom}_{\mathcal{F}}\left(Q,{ }^{h^{-1}} R\right)$ and that the homomorphism $\varphi=c_{g}: Q \rightarrow R$ factors as

$$
\varphi=c_{h} \circ \alpha: Q \rightarrow{ }^{h^{-1}} R \rightarrow R .
$$

Since ${ }^{h}(P, e)=(P, \gamma e)$, we obtain $h \in N_{G}(P, \tilde{e})$ and can write $h=g_{0}^{i} x$ for some $i \in \mathbb{Z}$ and $x \in N_{G}(P, e)$. This implies that the map $c_{h}: P \rightarrow P$ factors as $c_{h}=\sigma^{i} \circ \beta: P \rightarrow P$ where $\sigma^{i}=c_{g_{0}^{i}}: P \rightarrow P$ and $\beta:=c_{x} \in \operatorname{Aut}_{\mathcal{F}}(P)$, since $x \in N_{G}(P, e)$. Restriction to ${ }^{h^{-1}} R$ yields the factorization

$$
\left.c_{h}\right|_{h^{-1} R h}=\left.\left.\sigma^{i}\right|_{\beta\left(h^{-1} R h\right)} \circ \beta\right|_{h^{-1} R h}:{ }^{h^{-1}} R \rightarrow \beta\left({ }^{h^{-1}} R\right) \rightarrow R
$$

with $\beta\left({ }^{h^{-1}} R\right)=\sigma^{-i}(R)$ and $\left.\beta\right|_{h^{-1} R h} \in \operatorname{Hom}_{\mathcal{F}}\left({ }^{h^{-1}} R, R\right)$. Setting $\psi:=\left.\beta\right|_{h^{-1} R h} \circ \alpha: Q \rightarrow \sigma^{-1}(R)$ and using (3) we obtain the desired factorization of $\varphi$. This also implies the inclusion $\tilde{\mathcal{F}} \subseteq$ $\langle\mathcal{F}, \sigma\rangle$.

In order to find $\psi^{\prime}$ with the desired property we use the elements $g, h, x$, and $i$ from the first part of the proof and note that

$$
(P, e)={ }^{\gamma^{-1} h}(P, e) \geqslant_{L}{ }^{\gamma^{-1}}\left({ }^{h} Q,{ }^{h} e_{Q}\right)=\left({ }^{h} Q, \gamma^{-1} e_{Q}\right)
$$

which implies that $\gamma^{-1} h_{Q}=e_{h Q h^{-1}}$. Thus,

$$
{ }^{g h^{-1}}\left({ }^{h} Q, e_{h Q h^{-1}}\right)={ }^{g h^{-1}}\left({ }^{h} Q, \gamma^{-1} e_{Q}\right)=\left({ }^{g} Q,{ }^{g \gamma^{-1}} e_{Q}\right) \leqslant L\left(R, e_{R}\right),
$$

which implies that $\alpha^{\prime}:=c_{g h^{-1}}:{ }^{h} Q \rightarrow R$ belongs to $\operatorname{Hom}_{\mathcal{F}}\left({ }^{h} Q, R\right)$. Thus, $\varphi$ can be factored as

$$
\varphi=c_{g}=c_{g h^{-1}} \circ c_{h}=\alpha^{\prime} \circ c_{h}: Q \rightarrow{ }^{h} Q \rightarrow R .
$$

We can rewrite $h=g_{0}^{i} x=x^{\prime} g_{0}^{i}$ for some $x^{\prime} \in N_{G}(P, e)$ and obtain an element $\beta^{\prime} \in \operatorname{Aut}_{\mathcal{F}}(P)$ together with a factorization $c_{h}=\beta^{\prime} \circ \sigma^{i}: P \rightarrow P$. Restricting this equation to $Q$ yields a factorization

$$
c_{h}=\left.\left.\beta^{\prime}\right|_{\sigma^{i}(Q)} \circ \sigma^{i}\right|_{Q}: Q \rightarrow \sigma^{i}(Q) \rightarrow{ }^{h} Q .
$$

Setting $\psi^{\prime}:=\left.\alpha^{\prime} \circ \beta^{\prime}\right|_{\sigma^{i}(Q)} \in \operatorname{Hom}_{\mathcal{F}}\left(\sigma^{i}(Q), R\right)$, the factorization in (4) can now be expressed as $\varphi=\left.\psi^{\prime} \circ \sigma^{i}\right|_{Q}$ as claimed.

\section{Consequences of the Main Theorem}

In this section we prove several consequences of Theorem 5.2 .

Recall that if $\mathcal{F}$ is a fusion system over a $p$-group $P$, a subgroup $Q$ of $P$ is called $\mathcal{F}$-centric if $C_{P}(R)=Z(R)$ for all subgroups $R$ of $P$ which are $\mathcal{F}$-isomorphic to $Q$. 
6.1 Proposition Let $L / K, b,(P, e)$ and $\mathcal{F} \subseteq \tilde{\mathcal{F}}$ be as in Theorem 5.2.

(a) A subgroup $Q$ of $P$ is fully $\mathcal{F}$-centralized if and only if it is fully $\tilde{\mathcal{F}}$-centralized.

(b) A subgroup $Q$ of $P$ is fully $\mathcal{F}$-normalized if and only if it is fully $\tilde{\mathcal{F}}$-normalized.

(c) A subgroup $Q$ of $P$ is $\mathcal{F}$-centric if and only it is $\tilde{\mathcal{F}}$-centric.

Proof The 'if'-parts follow immediately from the fact that the $\mathcal{F}$-isomorphism class of $Q$ is a subset of the $\tilde{\mathcal{F}}$-isomorphism class of $Q$. For the forward implications note that by Theorem 5.2 two subgroups $Q$ and $Q^{\prime}$ of $P$ are $\tilde{\mathcal{F}}$-isomorphic if and only if there exists a subgroup $Q^{\prime \prime}$ of $P$ such that $Q$ is $\mathcal{F}$-isomorphic to $Q^{\prime \prime}$ and $Q^{\prime}=\sigma^{i}\left(Q^{\prime \prime}\right)$ for some $i \in \mathbb{Z}$. Moreover, $\sigma^{i}\left(C_{P}\left(Q^{\prime \prime}\right)\right)=C_{P}\left(\sigma^{i}\left(Q^{\prime \prime}\right)\right), \sigma^{i}\left(N_{P}\left(Q^{\prime \prime}\right)\right)=N_{P}\left(\sigma^{i}\left(Q^{\prime \prime}\right)\right)$, and $\sigma^{i}\left(Z\left(Q^{\prime \prime}\right)\right)=Z\left(\sigma^{i}\left(Q^{\prime \prime}\right)\right)$, since $\sigma^{i}$ is an automorphism of $P$. The result is now immediate.

The following Theorem is known to experts. See for instance the part of the proof of [L18, Theorem 8.5.2] dealing with the extension axiom and note that it does not use any assumptions on the field of coefficients $k$. Below is a proof with a different approach, using Theorem 5.2 .

6.2 Theorem Let $k$ be a field of characteristic $p>0$ and let $c$ be a block idempotent of $k G$. Then the extension axiom holds for the fusion system of $k G c$, for any choice of maximal Brauer pair.

Proof Let $(P, f)$ be a maximal $k G c$-Brauer pair. We apply Theorem 5.2 with $K=k$, a splitting field $L$ of $K C_{G}(P) f$ such that $L / K$ is a finite Galois extension with Galois group $\Gamma$, and to a block idempotent $b$ of $L G$ with $c b \neq 0$. Then $c=\tilde{b}$. Moreover, there exists a maximal $L G b$-Brauer pair $(P, e)$ such that $e f=e$ and therefore $f=\tilde{e}$. We aim to show that the fusion system $\tilde{\mathcal{F}}=\mathcal{F}_{(P, \tilde{e})}(K G \tilde{b})$ satisfies the extension axiom. Note that by Theorem 3.7 , the extension axoim holds for $\mathcal{F}=\mathcal{F}_{(P, e)}(L G b)$, since $L$ is a splitting field of $L C_{G}(P) e$. Let $\varphi \in \operatorname{Hom}_{\tilde{\mathcal{F}}}(Q, P)$ be such that $\varphi(Q)$ is fully $\tilde{\mathcal{F}}$-normalized. By Theorem 5.2 we can factorize $\varphi=\sigma^{i} \circ \psi$ for some $\psi \in \operatorname{Hom}_{\mathcal{F}}(Q, P)$. With $\varphi(Q)$ also $\psi(Q)=\sigma^{-i}(\varphi(Q))$ is fully $\tilde{\mathcal{F}}$-normalized, since they are $\tilde{\mathcal{F}}$-isomorphic and $N_{P}(\psi(Q))=\sigma^{-i}\left(N_{P}(\varphi(Q))\right)$. By Proposition 6.1(b), $\psi(Q)$ is fully $\mathcal{F}$-normalized. Since $\mathcal{F}$ satisfies the extension axiom, there exists $\hat{\psi} \in \operatorname{Hom}_{\mathcal{F}}\left(N_{\psi}, P\right)$ such that $\left.\hat{\psi}\right|_{Q}=\psi$. It follows that $\hat{\varphi}:=\sigma^{i} \circ \hat{\psi} \in \operatorname{Hom}_{\tilde{\mathcal{F}}}\left(N_{\psi}, P\right)$ extends $\varphi$. To finish the proof it suffices to show that $N_{\varphi} \subseteq N_{\psi}$. So let $x \in N_{\varphi}$. Then $x \in N_{P}(Q)$ and there exists $y \in N_{P}(\varphi(Q))$ with $\varphi \circ c_{x}=c_{y} \circ \varphi: Q \stackrel{\sim}{\rightarrow} \varphi(Q)$. But this implies

$$
\psi \circ c_{x}=\sigma^{-i} \circ \varphi \circ c_{x}=\sigma^{-i} \circ c_{y} \circ \varphi=c_{\sigma^{-i}(y)} \circ \sigma^{-i} \circ \varphi=c_{\sigma^{-i}(y)} \circ \psi,
$$

with $\sigma^{-i}(y) \in \sigma^{-i}\left(N_{P}(\varphi(Q))\right)=N_{P}\left(\sigma^{-i}(\varphi(Q))\right)=N_{P}(\psi(Q))$. Thus, $N_{\varphi} \subseteq N_{\psi}$ and the proof is complete.

6.3 Theorem Let $L / K, b,(P, e)$ and $\mathcal{F} \subseteq \tilde{\mathcal{F}}$ be as in Theorem 5.2, The fusion system $\tilde{\mathcal{F}}$ is saturated if and only if the fusion system $\mathcal{F}$ is saturated and $p$ does not divide $\left[N_{G}(P, \tilde{e})\right.$ : $\left.N_{G}(P, e)\right]=\left[\Gamma_{b}: \Gamma_{e}\right]=[K(e): K(b)]$. In particular, if moreover $L$ is a splitting field for $L C_{G}(P) e$, then $\tilde{\mathcal{F}}$ is saturated if and only if $p$ does not divide $\left[N_{G}(P, \tilde{e}): N_{G}(P, e)\right]=\left[\Gamma_{b}\right.$ : $\left.\Gamma_{e}\right]=[K(e): K(b)]$.

Proof Note that the map $N_{G}(P, e) \rightarrow \operatorname{Aut}_{\mathcal{F}}(P), g \mapsto c_{g}$ induces an isomorphism $N_{G}(P, e) / C_{G}(P) \rightarrow \operatorname{Aut}_{\mathcal{F}}(P)$ which maps $P C_{G}(P) / C_{G}(P)$ to $\operatorname{Aut}_{P}(P)$. Thus, the Sylow axiom holds for $\mathcal{F}$ if and only if $p \nmid\left[N_{G}(P, e): P C_{G}(P)\right]$. Similarly, the Sylow axiom holds for $\tilde{\mathcal{F}}$ if 
and only if $p \nmid\left[N_{G}(P, \tilde{e}): P C_{G}(P)\right]$. By Theorem 6.2 it suffices to show that the Sylow axiom holds for $\tilde{\mathcal{F}}$ if and only it holds for $\mathcal{F}$ and $p \nmid\left[\Gamma_{b}: \Gamma_{e}\right]$. But, by Proposition 5.1)(c), one has $\left[\Gamma_{b}: \Gamma_{e}\right]=\left[N_{G}(P, \tilde{e}): N_{G}(P, e)\right]=[K(e): K(b)]$ which implies the result.

Next we will show that a weak form of Alperin's fusion theorem holds for arbitrary block fusion systems.

6.4 Definition Let $\mathcal{F}$ be a fusion system over a $p$-group $P$. We say that Alperin's weak fusion theorem holds for $\mathcal{F}$ if $\mathcal{F}=\left\langle\operatorname{Aut}_{\mathcal{F}}(Q) \mid Q \in \mathcal{C}\right\rangle$, where $\mathcal{C}$ is the set of subgroups of $P$ which are $\mathcal{F}$-centric and fully $\mathcal{F}$-normalized.

6.5 Theorem Let $k$ be a field of characteristic $p$ and let $c$ be a block idempotent of $k G$. Then Alperin's weak fusion theorem holds for the fusion system of $k G c$, for any choice of maximal $k G c$-Brauer pair.

Proof Set $K:=k$ and choose $L, b,(P, e)$ as in the proof of Theorem 6.2 with $c=\tilde{b}$ and apply Theorem 5.2 to this situation with $\mathcal{F}:=\mathcal{F}_{(P, e)}(L G b)$ and $\tilde{\mathcal{F}}:=\mathcal{F}_{(P, \tilde{e})}(K G \tilde{b})$. We need to show that Alperin's weak fusion theorem holds for $\tilde{\mathcal{F}}$. Since $\mathcal{F}$ is saturated, Alperin's weak fusion theorem holds for $\mathcal{F}$, see for instance [L18, Theorem 8.2.8]. Thus, $\mathcal{F}=\left\langle\operatorname{Aut}_{\mathcal{F}}(Q)\right| Q \in$ $\mathcal{C}\rangle$, where $\mathcal{C}$ denotes the set of subgroups of $P$ which are $\mathcal{F}$-centric and fully $\mathcal{F}$-normalized. Moreover, by Proposition $6.1, \mathcal{C}$ is equal to the set $\tilde{\mathcal{C}}$ of subgroups of $P$ which are $\tilde{\mathcal{F}}$-centric and fully $\tilde{\mathcal{F}}$-normalized. Thus, by Theorem 5.2 , we have

$$
\tilde{\mathcal{F}}=\langle\mathcal{F}, \sigma\rangle=\left\langle\left\{\operatorname{Aut}_{\mathcal{F}}(Q) \mid Q \in \mathcal{C}\right\} \cup\{\sigma\}\right\rangle \subseteq\left\langle\operatorname{Aut}_{\tilde{\mathcal{F}}}(Q) \mid Q \in \mathcal{C}\right\rangle \subseteq \tilde{\mathcal{F}}
$$

But this implies $\tilde{\mathcal{F}}=\left\langle\operatorname{Aut}_{\tilde{\mathcal{F}}}(Q) \mid Q \in \mathcal{C}\right\rangle=\left\langle\operatorname{Aut}_{\tilde{\mathcal{F}}}(Q) \mid Q \in \tilde{\mathcal{C}}\right\rangle$, which means that Alperin's weak fusion theorem holds for $\tilde{\mathcal{F}}$.

\section{References}

[AKO11] M. Aschbacher, R. Kessar, B. Oliver: Fusion systems in Algebra and topology. London Mathematical Society Lecture Note Series, 391. Cambridge University Press, Cambridge, 2011.

[B10] S. Bouc: Biset functors for finite groups. Lecture Notes in Mathematics, 1990. SpringerVerlag, Berlin, 2010.

[L18] M. Linckelmann: The block theory of finite group algebras. Vol. II. London Mathematical Society Student Texts, 92. Cambridge University Press, Cambridge, 2018. 